\title{
Custom-made mouthguards protect football players
}

\author{
Do custom-made mouthguards really prevent injuries?
}

\author{
Finch C, Braham R, Mclntosh A, McCrory P, Wolfe R. \\ Should football players wear custom fitted mouthguards? Results \\ from a group randomised controlled trial. Inj Prev2005; 11:242- \\ 246.
}

Design A group randomised controlled trial (RCT) was conducted. Intervention Australian-rules football teams were randomly assigned to the test group (MG, custom-made mouthguards) or control group (usual mouthguard behaviour).

Outcome measure The number of head or orofacial $(\mathrm{H} / \mathrm{O})$ injuries per 1000 person-hours of playing was recorded.

Results Twenty-three Australian rules football teams were recruited of which 11 were randomised to the control group $(n=111)$ and 12 to the test group ( $n=190)$. The majority of players, including those in the control group, wore mouthguards during games; fewer wore them every training session. Overall rate of $\mathrm{H} / \mathrm{O}$ injuries was 2.7 per 1000 exposure$\mathrm{h}$, and was higher during games than training. When data were adjusted for division of play and age group there was evidence of a significant $(P=0.04)$ protective effect of $M G$ over control during games and training combined. The adjusted $\mathrm{H} / \mathrm{O}$ injury incidence rate ratio was 0.56 .

Conclusions Custom-made mouthguards provided a significant protective effect relative to usual mouthguard use during games.
Address for correspondence: Professor C Finch, New South Wales Injury Risk Management Research Centre, University of New South Wales, Sydney, NSW, Australia 2052. E-mail: c.finch@unsw.edu.au.

\section{Commentary}

It is widely accepted that sports mouthguards can provide protection for participants in contact sports. The level of evidence for their effectiveness is not high, however. Information has come mainly from retrospective surveys comparing orofacial injuries sustained by mouthguard users and non-users participating in various sports. These have generally, but not always, provided support of effectiveness. ${ }^{1}$

This well-designed study, part of a series investigating injuries in Australian-rules football, is the first prospective RCT testing the effectiveness of sports mouthguards. It has certainly demonstrated how difficult it is to conduct such an investigation. A major study design problem confronted by investigators is that it is unethical to ask players not to wear a mouthguard. All team members $(n=190)$ in the test group used thermoformed polyvinyl acetate-polyethylene (PVA/PE) custom-made mouthguards whilst playing and during training over the study period of a playing season. The team members in the control group ( $n=111)$, however, used or did not use mouthguards according to their usual practice. These used boil and bite mouthguards, custom-made guards or no mouthguards in an undetermined distribution and frequency for the period of the study. It was therefore as much a comparison group as a control group and, of course, greatly reduces the clarity of the findings.

A further difficulty of this study was that injuries were expressed as the H/O injuries per 1000 exposure-h. Head and orofacial injuries were considered together in the analysis of results in order to achieve sufficient statistical power. The central finding was that $\mathrm{H} / \mathrm{O}$ injuries in the test group were significantly fewer than in the control group.

The problems of the control arm and the grouping of injuries for analysis confuses some issues, but not the conclusion: this study provides good evidence of the potential effectiveness of custom-made PVA/PE mouthguards.

Practice point

- The study provides evidence supporting the effectiveness of custom-made PVA/PE mouthguards, when worn when playing or training, in reducing $\mathrm{H} / \mathrm{O}$ injuries.

\section{Jagger $\mathbf{R}$}

Department of Oral and Dental Science, Bristol Dental School, Bristol, UK.

1. Marshall SW, Loomis DP, Waller AE, et al. Evaluation of protective equipment for prevention of injuries in rugby union. Int J Epidemiol 2005; 34:113-118.

Evidence-Based Dentistry (2006) 7, 44.

doi:10.1038/sj.ebd.6400405 